\title{
On Net Stock Amplification in the Damped Trend Order-Up-To System
}

\author{
(presented at the 6th IESM Conference, October 2015, Seville, Spain) ${ }^{\circ} \mathrm{I}^{4} \mathrm{e}^{2} 2015$
}

\author{
Qinyun Li \\ Logistics Systems Dynamics Group, Logistics and \\ Operations Management Section, Cardiff Business School \\ Cardiff, United Kingdom \\ LiQY@ cardiff.ac.uk
}

\begin{abstract}
In this paper we characterize the frequency response of net stock amplification when the Damped Trend forecasting is used in the Order-Up-To replenishment policy. We prove that the invertibility regions from forecasting perspective are identical to the stability regions in control theory. From these stable and invertible regions, we explore the desirable parameter regions that the forecasting and inventory control policy is able to avoid the bullwhip effect and reduce net stock amplification for any lead-times. The simulations of 62 sets of real-world demand verify our analytical results.
\end{abstract}

Keywords-damped trend forecasting; order-up-to; net stock; bullwhip; invertibility; frequency response

\section{INTRODUCTION}

Since Damped Trend (DT) forecasting method was proposed [1], it has received considerable attentions in the literature. By dampening a linear trend, the method improves long-term forecast accuracy without significantly degrading short-term accuracy. A number of popular forecasting models such as Naïve, simple exponential smoothing, Holt's linear trend method can also be accessed by tuning the parameters of the general DT model. This allows for model detection and forecasting of a wide range of time series with or without trends. Its superior accuracy has been recognized in many empirical studies [2] [3] and academic reviews [4] [5] [6].

The DT method deserves more attention from supply chain management viewpoint. Economic benefits in terms of inventory, production, and shipping costs were reported in the applications in a real inventory scenario [7] and a real supply chain [8].

Reference [9] is the only one discussed the DT method in supply chain or operations management journals. They focused on the supply chain dynamics induced by the DT method, and found the bullwhip avoidance behavior that has never been seen before when other forecasting methods are used in the order-up-to (OUT) policy. The bullwhip effect is important in supply chains. It describes the scenario that the demand fluctuations are amplified from downstream to upstream throughout a supply chain. It creates severe consequences such as excessive capacity investment, inefficient use of transportation, labor idling and over-time.

\author{
Stephen Disney \\ Logistics Systems Dynamics Group, Logistics and \\ Operations Management Section, Cardiff Business School \\ Cardiff, United Kingdom \\ DisneySM@cardiff.ac.uk
}

In this paper, we focus on another important supply chain metrics, net stock amplification (NSAmp). NSAmp is related to the popular safety stock and fill rate concepts [10]. This paper also provides a proof that for the DT forecasting the invertibility regions and stability regions are identical, which means any stable DT forecasting model produces feasible forecasts. This allows us to study the model over the complete stability region from both control theory and forecasting perspectives

The paper is organized as follows: $\S 2$ introduces the model setup, $\$ 3$ discusses the invertibility of the DT method, $\$ 4$ investigates the NSAmp of the DT/OUT system, $\$ 5$ presents some simulation results and $\$ 6$ concludes.

\section{MODEL SETUP}

\section{A. Damped Trend Forecasting}

The single source of error correction form of Damped Trend forecasting is given by

$$
\begin{aligned}
& A_{t}=A_{t-1}+\phi B_{t-1}+\alpha \varepsilon_{t} \\
& B_{t}=\phi B_{t-1}+\alpha \beta \varepsilon_{t} \\
& \hat{D}_{t+k \mid t}=A_{t}+\sum_{i=1}^{k} \phi^{i} B_{t} .
\end{aligned}
$$

$\hat{D}_{t+k \mid t}$ is the forecast for $D_{t+k}$ made at time $t$. It is a product of $A_{t}$ (level) and $B_{t}$ (trend). $\{\alpha, \beta, \phi\}$ are systems parameters in the DT model.

The $z$-transfer functions of (1) are 


$$
\begin{aligned}
& \frac{A(z)}{\varepsilon(z)}=\frac{z^{2} \alpha+z \alpha \phi(\beta-1)}{z^{2}+z(\alpha-\phi-1+\alpha \beta \phi)+\phi(1-\alpha)} \\
& \frac{B(z)}{\varepsilon(z)}=\frac{z^{2} \alpha \beta-z \alpha \beta}{z^{2}+z(\alpha-\phi-1+\alpha \beta \phi)+\phi(1-\alpha)} \\
& \frac{\hat{D}_{k}(z)}{\varepsilon(z)}=\frac{z^{2} \alpha\left(1+\beta \sum_{i=1}^{k} \phi^{i}\right)+z \alpha\left(\phi(\beta-1)-\beta \sum_{i=1}^{k} \phi^{i}\right)}{z^{2}+z(\alpha-1-\phi+\alpha \beta \phi)+\phi(1-\alpha)} .
\end{aligned}
$$

For stability,

$$
\begin{gathered}
\phi-1<\alpha \phi<\phi+1 \\
\alpha(\phi-1)<\alpha \beta \phi<(2-\alpha)(\phi+1)
\end{gathered}
$$

are required [9].

\section{B. Damped Trend forecasting and Order-Up-To}

We follow the same model and assumptions in [9]. Let the replenishment lead-time from a manufacturer to a retailer, to be $T_{p} \in \aleph^{0}$ periods. In each period $t$, the retailer receives goods and satisfies demand $D_{t}$ from its on-hand inventory with complete backlogging of excess demand. Then, the retailer places order

$$
\begin{aligned}
O_{t} & =T N S+\hat{D}_{t+T_{p}+1 \mid t}+D W I P_{t}-\left(W I P_{t}+N S_{t}\right) \\
& =T N S+\hat{D}_{t+T_{p}+1 \mid t}+\underbrace{\sum_{i=1}^{T_{p}} \hat{D}_{t+i \mid t}}_{D W I P_{t}}-\underbrace{\sum_{i=1}^{T_{p}} O_{t-i}}_{W I P_{t}}-N S_{t}
\end{aligned}
$$

to the manufacturer. Net stock level

$$
N S_{t}=N S_{t-1}+O_{t-T_{p}-1}-D_{t}
$$

finalizes the model.

In (4), WIP is the work-in-progress, also known as inventory on-order. The time varying desired work-in-progress, $D W I P_{t}=\sum_{i=1}^{T_{p}} \hat{D}_{t+i \mid t}$ is the sum of the forecasts made at time $t$ for the periods from $t+1$ to $t+T_{p}$. TNS is a safety stock used to ensure a strategic level of inventory availability. Under the assumptions of normally distributed forecast errors and piecewise linear convex inventory holding $(h)$ and backlog costs $(b)$, it is common to assume $T N S=z \sigma_{N S} ; z=\Phi^{-1}\left[\frac{b}{b+h}\right]$. Here $\sigma_{N S}$ is the standard deviation of the net stock levels and $\Phi^{-1}[\bullet]$ is the inverse cumulative normal distribution function. $\sigma_{N S}$ is also related to the inventory costs. When $T N S=z \sigma_{N S}$, the expected inventory costs per period is
$J_{£}=\sigma_{N S}(b+h) \varphi[z]$, where $\varphi[z]$ is the probability density function of the normal distribution evaluated at $z$.

The net stock transfer function is

$$
\frac{N S(z)}{\varepsilon(z)}=\left(1-z^{-1}\right)^{-1}\left(z^{-T_{p}-1} \frac{O(z)}{\varepsilon(z)}-1\right)
$$

where $O(z) / \varepsilon(z)$ is the $z$-transfer function of orders. The close form of (6) is too large and complex to be presented at here, while all the necessary transfer functions including the order transfer function for the DT/OUT model have been documented in [9]. We found that the denominators of the order and net stock transfer functions have the same two poles as the transfer function of the DT forecasting system (2). This implies that the same stability conditions hold for the DT/OUT system as for the DT forecasting system.

We assume that both the retailer and the manufacturer use the OUT policy. Negative order quantities are allowed, but average demand is sufficiently large so that the probability of negative demand or negative orders is negligible.

\section{INVERTIBILITY}

The concept of invertibility in time series analysis refers to the feasibility of the identification of the demand process structure from past observations of demand. Invertibility is related to linear moving average (MA) models or the MA part of autoregressive integrated moving average (ARIMA) models. If an MA model (or an MA part in ARIMA models) can be expressed as an autoregressive (AR) model of infinite order, the model is deemed invertible, and implies all relevant state variables are directly observable. By investigating the arithmetic relationship between stability and invertibility for the DT forecasting system, we found the invertibility region is the same as the stability region for DT method.

All linear exponential smoothing methods have equivalent ARIMA models [11]. The pure DT method is equivalent to the $\operatorname{ARIMA}(1,1,2)$ model, which can be written as

$$
(1-B)(1-\phi B) d_{t}=\underbrace{\left(1-\theta_{1} B-\theta_{2} B^{2}\right)}_{\theta(B)} e_{t}
$$

where B is backward shift operator (similar to but not quite the same as $z^{-1}$ in control theory), $\theta_{1}=1+\phi-\alpha-\alpha \beta \phi$ and $\theta_{2}=\alpha \phi-\phi[12]$. For the second-order MA part, it is invertible only if the roots of the characteristic equation

$$
\theta(B)=1-\theta_{1} B-\theta_{2} B^{2}=0
$$

lie outside the unit circle. 
Li, Q. and Disney, S.M., (2015), "On net stock amplification in the Damped Trend Order-Up-To system",

6th International Conference on Industrial Engineering and Systems Management, Seville, Spain, 21s $23^{\text {rd }}$ October.

In fact, the denominator of the DT forecasting transfer function (2) can be rewritten using $\theta_{1}$ and $\theta_{2}$ as $z^{2}-\theta_{1} z-\theta_{2}$. The stability condition in control theory requires the roots of

$$
z^{2}-\theta_{1} z-\theta_{2}=0
$$

lie inside the unit circle.

It is easy to notice that the roots of (8) are the inverse of the roots of (9). Remember the test for stability requires the roots of (9) be inside the unit circle, invertibility requires the roots of (8) to be outside the unit circle. So the results for invertible regions and stable regions should be identical. Or solving for the roots of (8) to find the invertibility, we obtain

$$
H_{1,2}=\left(\theta_{1} \pm \sqrt{\theta_{1}^{2}+4 \theta_{2}}\right) / 2
$$

There are two possible pairs of values, but this is not a problem. Solving the simultaneous inequalities $\left|H_{i}\right|<1$ for $i=1,2$, both pairs give rise to identical calculations. The results are the same as our stability conditions in (3) when $\{\alpha, \beta\} \in \mathfrak{R}$. Jury's Inners approach [13] can also be used to study the invertibility region, and also returns the exactly same regions as the stability regions.

Thus, we conclude the invertibility regions are the same as the stability regions for DT forecasting, even though the implications of stability and invertibility are different. The consistency between stability regions and invertibility regions becomes important, because now any stable DT parameter sets produce feasible forecasts. Therefore, it is interesting to investigate the system performance over the complete stability region.

\section{NET STOCK AMPLIFICATION WITH BULLWHIP AVOIDANCE PARAMETERS}

\section{A. Desirable Parameter Values}

Traditional suggestions advocate to restrict $\{\alpha, \beta, \phi\}$ to the $[0,1]$ interval. However, we found in the previous section the DT forecasting is invertible and stable over a wide range of parameter values. In addition, [9] proved that the bullwhip avoidance behavior only occurs with some unconventional but stable $\{\alpha, \beta, \phi\}$. They proposed a range of values that are able to reduce the bullwhip ratio to less than one.

We extended their bullwhip analysis from $T_{p}=1$ to different lead-time (details omitted for brevity), and found that:

- The parametrical plane $\phi>1$ still can enable the DT/OUT system to avoid the bullwhip effect.

- For $0<\phi<1$, there is no change in the criteria $\{\alpha, \phi\}$ and the upper boundary of $\beta$. Only the lower boundary of $\beta$ value increases with the lead-time (see Fig. 1).

Table 1 details the bullwhip avoidance region when $0<\phi<1$ for different lead-times. Because the upper boundary of $\beta, \frac{\phi-1}{\phi}<0$, it is easy to conclude when $0<\phi<1$ the area of the parametrical plane that is able to avoid the bullwhip effect becomes smaller if the leadtime increases.

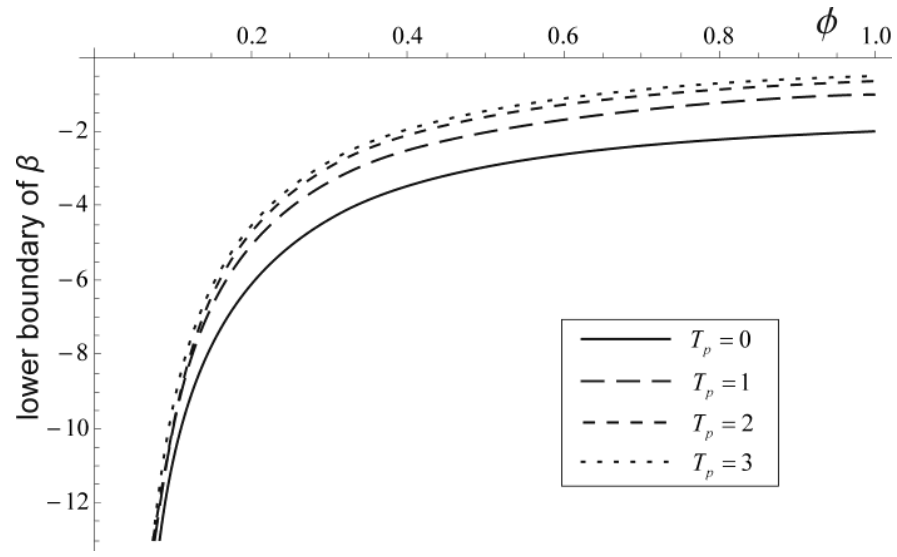

Fig. 1. Lower boundary of $\beta$ changes with $T_{p}$

TABLE I. BULlWhIP AVOIDANCE AREA $0<\phi<1$ FOR DIFFERENT LEAD-TIMES

\begin{tabular}{|l|c|}
\hline$T_{p}$ & $0<\phi<1, \frac{\phi-1}{\phi}<\alpha<0$, lower boundary $<\beta<\frac{\phi-1}{\phi}$ \\
\hline 0 & $\frac{-1-\phi}{\phi}$ \\
\hline 1 & $\frac{-1}{\phi}$ \\
\hline 2 & $\frac{-3-3 \phi}{3 \phi+4 \phi^{2}+2 \phi^{3}}$ \\
\hline 3 & $\frac{-2}{2 \phi+\phi^{2}+\phi^{3}}$ \\
\hline 4 & $\frac{-5-5 \phi}{5 \phi+8 \phi^{2}+6 \phi^{3}+4 \phi^{4}+2 \phi^{5}}$ \\
\hline 5 & $\frac{-3}{3 \phi+2 \phi^{2}+2 \phi^{3}+\phi^{4}+\phi^{5}}$ \\
\hline 6 & $\frac{-7-7 \phi}{7 \phi+12 \phi^{2}+10 \phi^{3}+8 \phi^{4}+6 \phi^{5}+4 \phi^{6}+2 \phi^{7}}$ \\
\hline 7 & $\frac{-4}{4 \phi+3 \phi^{2}+3 \phi^{3}+2 \phi^{4}+2 \phi^{5}+\phi^{6}+\phi^{7}}$ \\
\hline 8 & $\frac{-9-9 \phi}{5 \phi+16 \phi^{2}+14 \phi^{3}+12 \phi^{4}+10 \phi^{5}+8 \phi^{6}+6 \phi^{7}+4 \phi^{8}+2 \phi^{9}}$ \\
\hline 9 & $\frac{-5}{5 \phi+3 \phi^{4}+3 \phi^{5}+2 \phi^{6}+2 \phi^{7}+\phi^{8}+\phi^{9}}$ \\
\hline
\end{tabular}

- The region within the parametrical plane $-1<\phi<0$ where bullwhip is avoided changes in a complex manner. It has different shapes when the lead-time changes from odd to even. 
Li, Q. and Disney, S.M., (2015), "On net stock amplification in the Damped Trend Order-Up-To system",

6th International Conference on Industrial Engineering and Systems Management, Seville, Spain, 21s $23^{\text {rd }}$ October.

- Any bullwhip avoiding areas of the parametrical plane $\phi<-1$ disappears and reappears in sophisticated manners when the lead-time switches between an odd number and an even number.

The bullwhip avoidance areas when $\phi>1$ or $0<\phi<1$ are less influenced by the lead-time compared to other planes. As the parametrical plane $0<\phi<1$ enables the amplification for only a very few frequencies [9], which outperforms $\phi>1$, this paper mainly focuses on the performance when the value of DT parameters are selected from the bullwhip avoiding region $0<\phi<1$. When $T_{p}=1$, this area is reduced to $\{0<\phi<1,((\phi-1) / \phi)<\alpha<0,(-1 / \phi)<\beta<((\phi-1) / \phi)\}$.

\section{B. Analyzing Net Stock Amplification}

NSAmp usually is measured by the ratio of variance of net stock over the variance of demand. For independently and identically distributed (i.i.d.) demand, by taking the sum of the squared impulse response from (6), we have

$$
\begin{aligned}
& (\phi-1)^{4}(1+(\alpha-1) \phi)(\alpha-2+(\alpha+\alpha \beta- \\
& 2) \phi) \alpha\left(1-T_{p}^{2}(\phi-1)^{2}(1+(\beta-1) \phi)(\phi(1-\right. \\
& \left.\left.\alpha+\phi-2 \alpha \beta \phi+(\alpha-1) \phi^{2}\right)-1\right)+\phi(5 \phi- \\
& 4+\beta(\phi-1)^{4}(1+\phi)-\phi^{3}(5+(\phi-4) \phi)+ \\
& \alpha\left(1+\phi\left(\phi(2+(\phi-2)(\phi-1) \phi)+2 \beta^{2} \phi^{2}\right.\right. \\
& \left(\phi^{T_{p}}-1\right)\left(1-2 \phi+\phi^{2+T_{p}}\right)-3+\beta(\phi-1)^{2} \\
& \left.\left.\left.\left(1+\phi\left(2 \phi^{T_{p}}(1+\phi)-\phi-4\right)\right)\right)\right)\right)-2 T_{p}(\phi- \\
& 1)(1+(\beta-1) \phi)(1+\phi(\alpha(1+\phi((\phi-1) \phi- \\
& \left.\left.1+\beta\left(1+\phi\left(\phi^{T_{p}}(\phi+1)-3\right)\right)\right)\right)- \\
& \frac{\sigma_{N S}^{2}}{\sigma_{D}^{2}}=1+T_{p}-\frac{\left.\left.\left.(\phi-2) \phi^{2}\right)\right)\right)}{(\phi-1)^{4}(1+(\alpha-1) \phi)((\alpha-2)(1+\phi)+\alpha \beta \phi)} .
\end{aligned}
$$

Equation (11) provides an interesting suggestion that it might be possible to achieve $\sigma_{N S}^{2} \leq\left(1+T_{p}\right) \sigma_{D}^{2}$. This is a new finding considering that the OUT policy with other forecasting methods always require that $\sigma_{N S}^{2}>\left(1+T_{p}\right) \sigma_{D}^{2}$ for i.i.d. demand [14]. They show that the first unit of inventory variance is because of the order of events. From (11) it is easy to spot this inherent behavior as well. However, unlike the situations when other forecasting methods are used in the OUT policy, the DT method sometimes produces a negative function after $1+T_{p}$, therefore enabling the OUT policy to create less inventory variance. That means when there is no lead-time, inventory levels is able to vary less than the variation in the demand signal. In other words, it is possible to achieve a near zero inventory policy.

For a general case of demand, we can study the frequency response of the net stock variance. Let the modulus of the transfer function be denoted by Amplitude Ratio $(A R)$. Reference [9] characterized the frequency response plots for orders. Based upon that, they investigated the bullwhip creating or avoidance behavior of systems for arbitrary demand.

Assume $\left|\frac{N S\left(e^{j \omega}\right)}{\varepsilon\left(e^{j \omega}\right)}\right|=A R_{N S}$, then the initial value

$$
\left.A R_{N S}\right|_{\omega \rightarrow 0}=0
$$

as $\lim _{\omega \rightarrow 0} A R_{N S}=0$. The initial value suggests that for lowfrequencies, the DT/OUT system is possible to avoid creating net stock variance amplification.

The final value is

$$
\left.A R_{N S}\right|_{\omega=\pi}=\frac{\mid \begin{array}{l}
\left(-2\left(1+(-1)^{T_{p}}\right)(\phi-1)^{2}(\phi+1)+\alpha\left((-1)^{T_{p}}-1+\right.\right. \\
2 T_{p}(1+(\beta-1) \phi)\left(\phi^{2}-1\right)+\phi\left(\left((-1)^{T_{p}}-1\right)\right. \\
((\phi-1) \phi-1)+\beta\left((-1)^{T_{p}}-1+\phi\left(2-2(-1)^{T_{p}}+\right.\right. \\
\left.\left.\left.\left.\phi\left(3+(-1)^{T_{p}}-4 \phi^{T_{p}}\right)\right)\right)\right)\right)
\end{array}}{2(\phi-1)^{2}(2-\alpha+(2-\alpha-\alpha \beta) \phi)} .
$$

When $T_{p}=1,(13)$ is simplified to

$$
\left.A R_{N S}\right|_{\omega=\pi}=\frac{2 \alpha(1+\phi)(1+\beta \phi)}{\alpha+\alpha \phi+\alpha \beta \phi-2 \phi-2}
$$

Equation (14) is smaller than one if the parameters' values are from the bullwhip avoidance area in [9]. This is suggesting that for the demand dominated by high-frequency harmonics, such as the AR(1) demand with i.i.d. noise when the demand correlation is negative, once the DT/OUT system can avoid creating the bullwhip, most likely it would also reduce the amplification in net stock variance.

Fig. 2 illustrates an example of the frequency response plot of net stock. It acts as a low-pass filter, in which the lowfrequency harmonic magnitudes will be amplified, but NSAmp will be avoided when high-frequency harmonics dominate the demand. Note that with this setting, the DT/OUT system also acts as a low-pass filter in terms of orders [9].

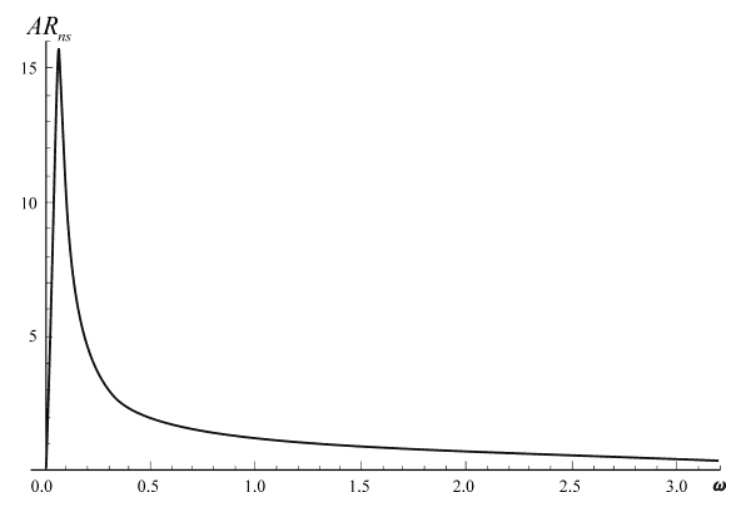

Fig. 2. AR plot of NSAmp when $\phi=0.5, \alpha=-0.9, \beta=-1.01, T_{p}=1$ 
Li, Q. and Disney, S.M., (2015), "On net stock amplification in the Damped Trend Order-Up-To system",

6th International Conference on Industrial Engineering and Systems Management, Seville, Spain, 21sŁ23rd October.

More importantly, for general lead-time cases, the final value of $A R_{N S}$ is always less than unity as long as the $\{\alpha, \beta, \phi\}$ is selected from Table 1 . These suggest the bullwhip avoidance parameter settings given by $\left\{0<\phi<1, \frac{\phi-1}{\phi}<\alpha<0\right\}$ probably have a desirable frequency response in terms of both orders and net stock levels for any lead-time.

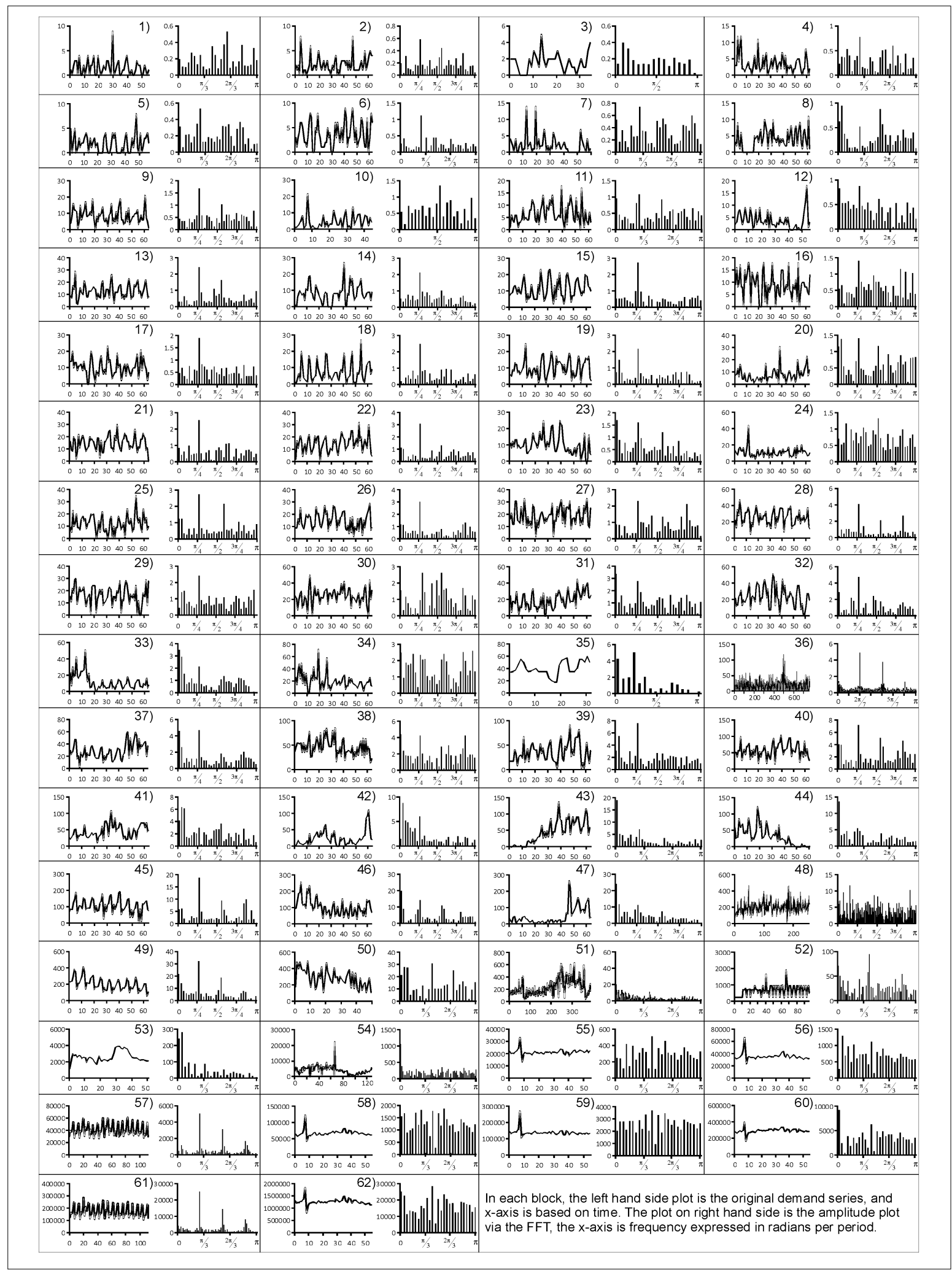

Fig. 3. Illustration of 62 real-world data sets 


\section{NUMERICAL SIMULATIONS}

In this section, 62 sets of real-world demand data are applied to verify our analytical results. These demand series came from previous research and projects conducted by the members of Logistics Systems Dynamics Group, Cardiff Business School. There are low-volume products as well as high volume products. The source of the data ranges from retailers, manufacturers, to logistics companies and distributors. Intermittent demand series are excluded.

These 62 real-world demand series are first decomposed into frequency harmonics by the Fast Fourier Transform (FFT). Fig. 3 illustrates each data set in both the time and frequency domain via a plot of amplitudes obtained from FFT. It is easy to notice that the majority of them are dominated by lowfrequency harmonics, except for series 55, 56, 58, 59 and 62 .

We assume the lead-time $T_{p}=1$, which matched the reallife scenario. In order to reduce the computing time, we narrowed down the search range to the desirable region $\left\{0<\phi<1, \frac{\phi-1}{\phi}<\alpha<0, \frac{\phi-1}{\phi}<\beta<\frac{-1}{\phi}\right\}$. This is also because within this area the DT/OUT system is most likely to have both good bullwhip and NSAmp as we explored in $\S 4$.

Numerical optimization based on minimizing $\sigma_{O}$ is first explored. This objective is to minimize the standard deviation of the orders, in other words, it is able to reduce the bullwhip effect. It is easy to observe from Fig. 4 that the OUT policy with DT forecasting mechanism successfully avoids the bullwhip effect in all the 62 time series. This is in accordance with [9] that the DT/OUT system is able to avoid the bullwhip effect with unconventional parameter values. However, the bullwhip avoidance sometimes came at the cost of a large amplification in net stock variance.

Despite the fact that some bullwhip ratios are close to zero, the bullwhip performance cannot achieve the same level as the model advocated by [15] that a proportional OUT policy can reduce order variance (and hence the bullwhip ratio) down to zero.

The second objective is to minimize the standard deviation of net stock. It is closely related to the safety stock a company must hold in inventory to minimize holding and backlog costs. Therefore, a minimized $\sigma_{N S}$ is able to reduce inventory costs and will also improve service level for a given safety stock.

When the objective function is to minimize $\sigma_{N S}$ (Fig. 5), the majority of NSAmp ratios were around two, and the NSAmp ratios were reduced to less than two in 17 of 62 real-world data sets. This verifies our previous finding that it is possible to achieve $N S A m p \leq 1+T_{p}$ (here in the simulations the lead-time $\left.T_{p}=1\right)$.

The parameter settings within that area can still eliminate the bullwhip effect in 35 of the 62 real-world data sets. For those scenarios which cannot avoid bullwhip, the majority of the bullwhip ratios were still maintained near one. This might suggest that the DT / OUT system can keep good control of inventory costs and service levels without inducing significant capacity costs. Managers only need to consider minimizing the standard deviation of inventory when inventory costs are more significant than capacity costs in their supply chains, as this will simultaneously generate the best inventory performance and relatively good bullwhip behavior.

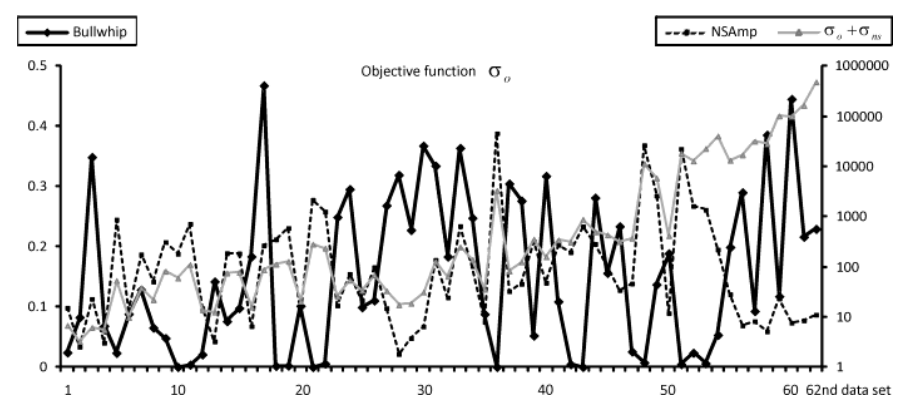

Fig. 4. The bullwhip and NSAmp when the objective is to minimize $\sigma_{O}$

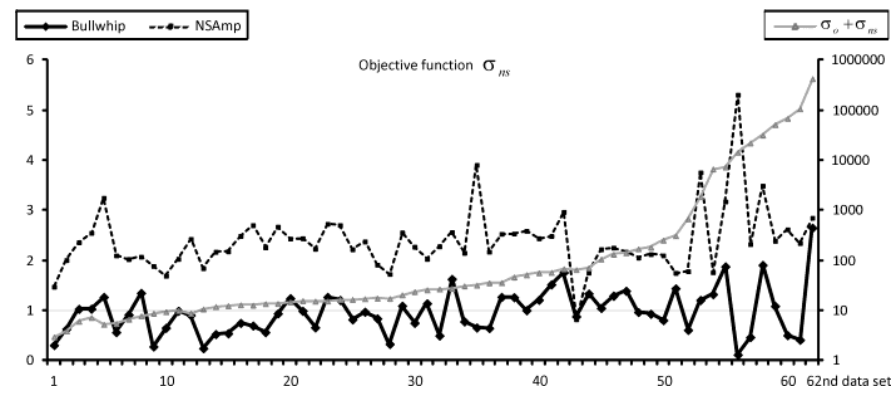

Fig. 5. The bullwhip and NSAmp when the objective is to minimize $\sigma_{N S}$

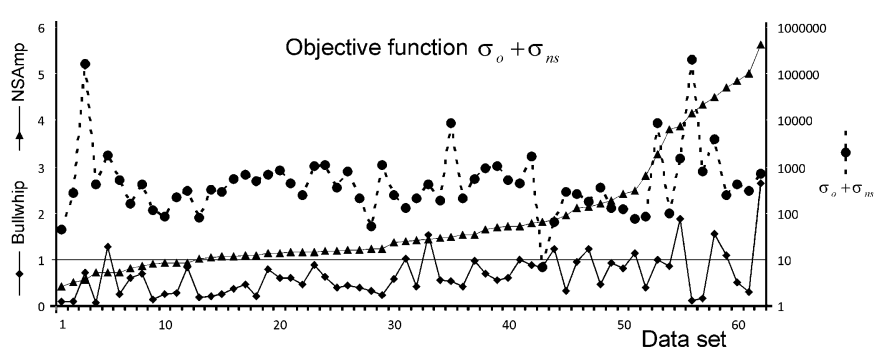

Optimised parameters

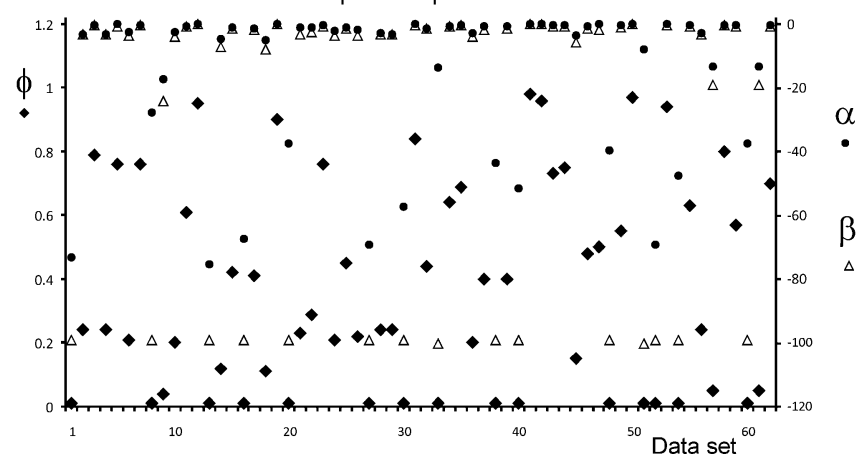

Fig. 6. The bullwhip and NSAmp of 62 real-world data sets when $\sigma_{O}+\sigma_{N S}$ is minimized, together with the optimized DT parameters

The last objective is to minimize $\sigma_{O}+\sigma_{N S}$ which considers the situation that a company might focus on both the capacity costs and inventory costs. Then we arbitrarily assume the 
Li, Q. and Disney, S.M., (2015), "On net stock amplification in the Damped Trend Order-Up-To system",

6th International Conference on Industrial Engineering and Systems Management, Seville, Spain, 21s $23^{\text {rd }}$ October.

standard deviation of inventory and the standard deviation of orders are equally costly.

If inventory costs and capacity costs are considered equally important, the empirical results show that the DT / OUT system successfully eliminated the bullwhip effect in 52 out of 62 time series when the objective is to $\min \left(\sigma_{O}+\sigma_{N S}\right)$ (Fig. $6)$. In the majority of the cases, the NSAmp ratios were maintained between one and three.

Observing the optimized parameters, we notice that only a few optimized $\phi$ values are close to one. The majority of the optimized $\alpha$ and $\beta$ have less negative values, which is consistent with the recommendation in [9].

\section{CONCLUSIONS}

Via 62 real-world data sets, this paper has verified the findings in [9] that the Damped Trend forecasting is able to avoid the bullwhip effect when it is used in the Order-Up-To policy. Its bullwhip avoidance behavior exists even with different objective functions. We also provided the analytical expressions of the desirable parameter region for the bullwhip avoidance with different lead-times.

This paper is more than an extension of [9]. By proving the invertibility regions and the stability regions of the DT forecasting are identical, we offered the theoretic support to exploring the performance of the DT/OUT system over a wider range of parameter values than conventional recommendations. This is important both theoretically and practically, because if the bullwhip can be avoided, unconventional parameter values must be selected [9].

More importantly, the analytical and simulation studies in this paper have shown that the DT/OUT system is also able to reduce the net stock amplification, further down to less than lead-time plus one. Using the bullwhip avoidance parameters, the DT/OUT system is most likely to reduce the net stock amplification. Thus, it is worth to consider applying the parameter values from the desirable region we proposed. The superior inventory control that the DT/OUT system exhibits, the ability of avoid inducing significant capacity costs, make the DT/OUT system a good choice for organizations emphasizing inventory costs and service levels.

This paper took an alternative route to consider forecasting in supply chains. The majority of studies in the academic literature that consider forecasting and inventory control tend to ignore the effect of using estimated forecasting parameters [16]. For instance, traditional forecasting practice would treat the demand series 9 in Fig. 3 as a series without trend. Thus, $\phi=0$ is more suitable to minimize forecast errors. Then, the forecasting and inventory control system will definitely create the bullwhip effect in supply chains [9]. In the paper, forecasts were optimized on the performance of order and/or inventory performance. By doing so, the optimized $\phi$ value is 0.04 and both $\alpha$ and $\beta$ are negative. Then, it is possible to reduce the bullwhip ratio to 0.13 , and net stock amplification ratio to 2.05 , when we are optimizing $\sigma_{O}+\sigma_{N S}$. Less bullwhip and less net stock amplification concur with business's interests in production planning and inventory control better than forecast accuracy does, because focusing on forecasting accuracy in this case will never achieve the same level of performance.

However, forecasting is still important for other diagnostic purposes. The paper can be improved by considering the forecasting performance when the bullwhip effect is avoided and the net stock amplification is reduced. Performance measures such as service level can also be included in future research.

\section{REFERENCES}

[1] E.S. Gardner Jr. and E. McKenzie, "Forecasting trends in times series," Manage. Sci., vol. 31, pp. 1237-1246, October 1985.

[2] S. Makridakis and M. Hibon, "The M3-competition: results, conclusions and implications," Int. J. Forecast., vol. 16, pp. 451-476, OctoberDecember 2000.

[3] E.S. Gardner Jr. and J. Diaz-Saiz, "Exponential smoothing in the telecommunications data," Int. J. Forecast., vol. 24, pp. 170-174, January-March 2008.

[4] J.S. Armstrong, "Findings from evidence-based forecasting: methods for reducing forecast error,” Int. J. Forecast., vol. 22, pp. 583-598, 2006.

[5] R. Fildes, K. Nikolopous, S.F. Crone, and A.A. Syntetos, "Forecasting and operational research: a review," J. Oper. Res. Soc., vol. 59, pp.11501172, September 2008.

[6] E.S. Gardner Jr. and E. McKenzie, "Why the damped trend works," J. Oper. Res. Soc., vol. 62, pp. 1177-1180, June 2011.

[7] E.S. Gardner Jr., "Evaluating forecasting performance in an inventory control system," Manage. Sci., vol. 36, pp. 490-499, April 1990.

[8] Y. Acar and E.S. Gardner Jr., "Forecasting method selection in a global supply chain,” Int. J. Forecast., vol. 28, pp. 842-848, October-December 2012.

[9] Q. Li, S.M. Disney, and G. Gaalman, "Avoiding the bullwhip effect using Damped Trend forecasting and the Order-Up-To replenishment policy," Int. J. Prod. Econ., pp. 3-16, March 2014.

[10] P.H. Zipkin, Foundations of Inventory Management. New York: McGraw-Hill, 2000.

[11] G.E.P. Box, G.M. Jenkins, and G.C. Reinsel, Time Series Analysis: Forecasting and Control. New Jersey: John Wiley \& Sons, 2008.

[12] S.A. Roberts, "A general class of Holt-Winters type forecasting models,” Manage. Sci., vol. 28, pp. 808-820, July 1982.

[13] E.I. Jury, "Inners approach to some problems of system theory," IEEE Trans. Autom. Control, vol. 16, pp. 233-240, June 1971.

[14] S.M. Disney and D.R. Towill, "On the bullwhip and inventory variance produced by an ordering policy," Omega, vol. 31, pp. 157-167, June 2003.

[15] J. Dejonckheere, S.M. Disney, M.R. Lambrecht, and D.R. Towill, "Measuring and avoiding the bullwhip effect: a control theoretic approach,” Eur. J. Oper. Res., vol. 147, pp. 567-590, June 2003.

[16] L.W.G. Strijbosch, A.A. Syntetos, J.E. Boylan, and E. Janssen, "On the interaction between forecasting and stock control: the case of nonstationary demand,” Int. J. Prod. Econ., pp. 470-480, September 2011. 